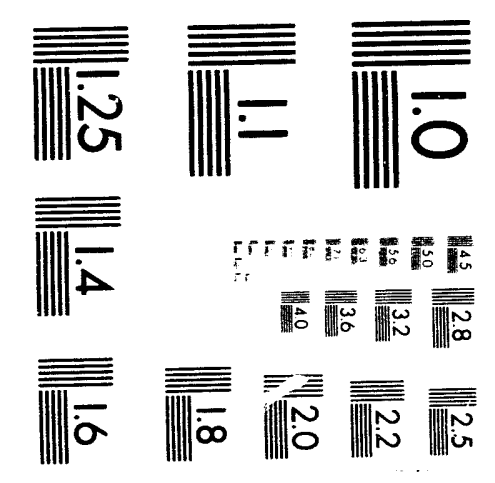



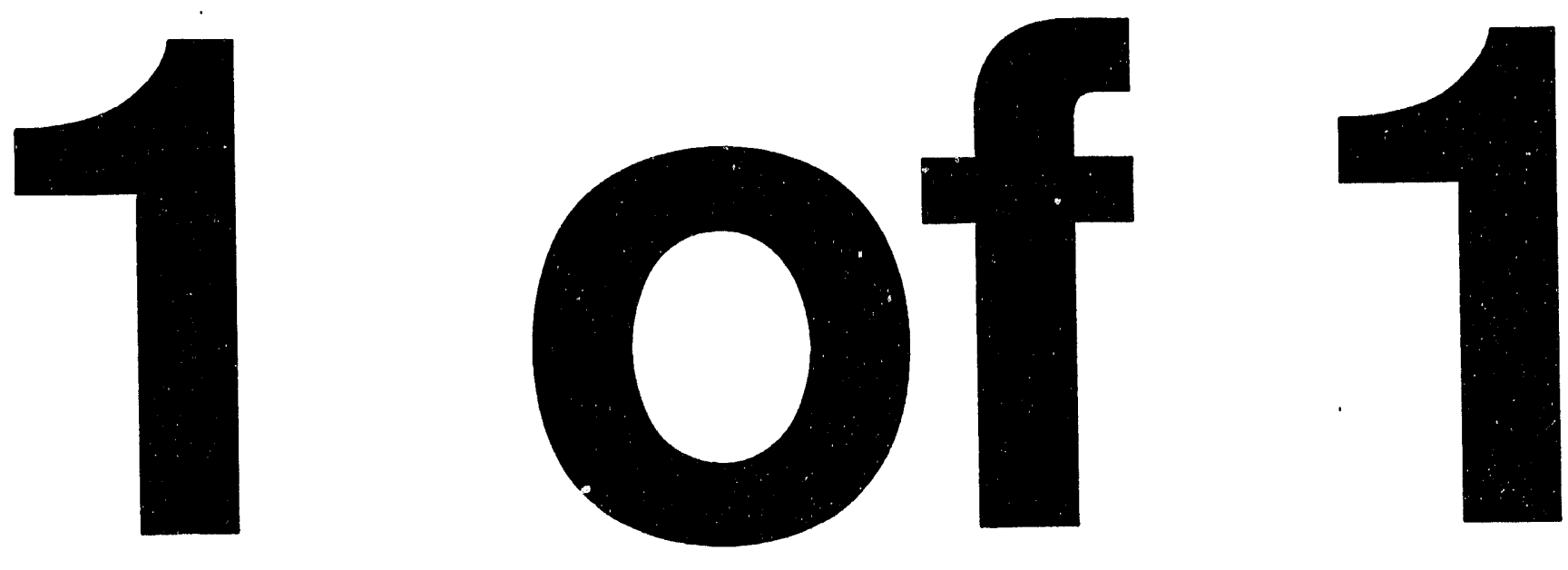


\title{
REVIEW OF \\ TOKAMAK EXPERIMENTS ON DIRECT ELECTRON HEATING AND CURRENT DRIVE WITH FAST WAVES
}

\author{
by \\ R.I. PINSKER
}

This is a preprint of an invited paper presented at the Tenth Topical Conference on Radio Frequency Power in Plasmas, April 1-3, 1993, Boston, Massachusetts, and printed in the Proceedings.

\author{
Work supported by \\ U.S. Department of Energy \\ Contract DE-AC03-89ER51114
}

GENERAL ATOMICS PROJECT 3466 DECEMBER 1993

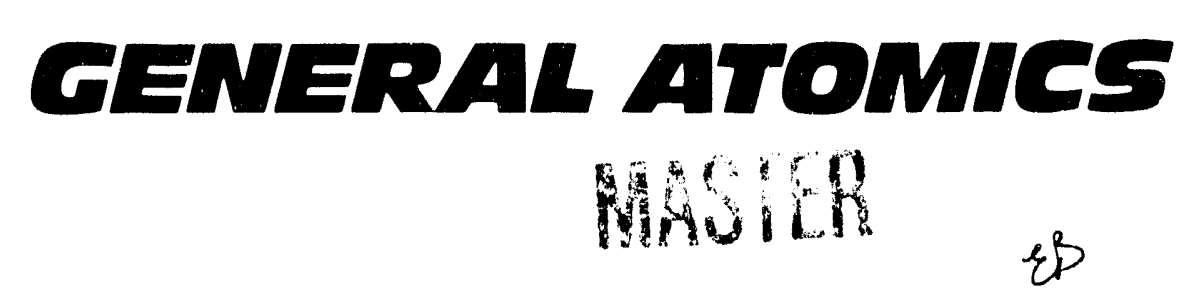




\title{
REVIEW OF TOKAMAK EXPERIMENTS ON DIRECT ELECTRON HEATING AND CURRENT DRIVE WITH FAST WAVES
}

\author{
Robert I. PinsKer \\ General Atomics, San Diego, CA 92186-9784
}

\section{ABSTRACT}

Results from tokamak experiments on direct electron interaction with the compressional Alfvén wave ('fast wave') are reviewed. Experiments aimed at electron heating as well as those in which fast wave electron current drive was investigated are discussed. A distinction is drawn between experiments employing the lower hybrid range of frequencies, where both the lower hybrid wave ('slow wave') and the fast wave can propagate in much of the plasma, and those experiments using the fast wave in the range of moderate to high ion cyclotron harmonics, where only the fast wave can penetrate to the plasma core. Most of the early tokamak experiments were in the lower hybrid frequency regime, and the observed electron interaction appeared to be very similar to that obtained with the slow wave at the same frequency. In particular, electron interaction with the fast wave was observed only below a density limit nearly the same as the well known slow wave density limit. In the more recent lower frequency fast wave experiments, electron interaction (heating and current drive) is observed at the center of the discharge, where slow waves are not present.

\section{INTRODUCTION}

Over the past two decades, the heating of tokamak plasmas by injection of compressional Alfvén waves ('fast waves') has become a mature subject (see Ref. 1 for an interesting review of some aspects of this development). In almost all of these experiments, the mechanism for absorption of the wave energy is ion cyclotron damping, at either the fundamental resonance of a minority ion species $\left(\omega=\Omega_{\text {mis }}\right) O_{s}$ at a low order harmonic of the majority $\left(\omega=n \Omega_{\mathrm{maj}}, n=2\right.$ or 3 ). In recent yea $s$, an alternative mechanism for fast wave absorption in a collisionless plasma, the coberent combination of electron Landau damping and transit time damping, has bsen explored. in a number of tokamak experiments. This absorption mechanism was d i ussed along with absorption at cyclotron resonances by Stix. ${ }^{2}$ An important appli ution of this method of interacting with electrons in a tokamak discharge is unir dictive ci crent drive; by transferring toroidal momentum from the fast wave to the ele. ons, at roidal

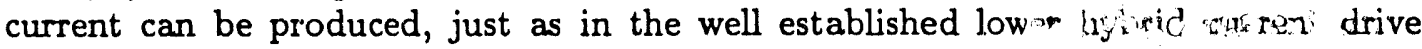

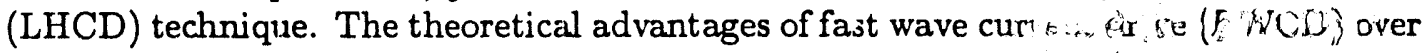
LHCD in a tokamak reactor are well known: no density limit ellon intsion at a given FW frequency, excellent accessibility to the plaspict $\mathrm{sc}$ is igh $\beta$, high $T_{e}$ discharges, current drive efficiencies equal to or greater

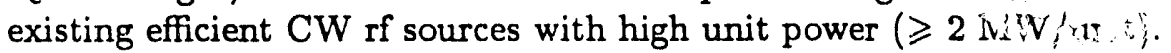

Non-inductive current drive is not the only use for direct $x=2 .+4$ tion with fast waves. Another important application to the tokamak is $s$. . . run heating method with several unique properties: the damping will always b.: scrongly peaked at the point of maximum electron $\beta$, usually near the magnetic axis, and tha power deposition profile shape will be nearly independent of the toroidal field and the density profile shape. Furthermore, since the power is not transferred to the electrons via an 
energetic ion tail, as is the case for low field side ICRH and for NBI, the power coupled to the electrons is not limited by fast ion energetics ${ }^{3}$ or instabilities (TAE, fishbones, etc.).

The fast wave exists in a magnetized plasma over a very wide range of frequencies, from the MHD $\left(\omega \ll \Omega_{\mathrm{i}}\right)$ regime, where it is usually referred to as the compressional Alfvén wave*, through the ion cyclotron range of frequencies and the lower hybrid range of frequencies. While FWCD may be applied in a tokamak reactor at a frequency just below the lowest ion cyclotron resonance frequency to avoid damping on alpha particles, the lowest frequency regime of fast wave propagation has not yet been the subject of many experimental studies at high power levels in tokamaks, and therefore will not be discussed further in this review. Most of the physics of direct electron interaction with the fast wave just below $\Omega_{\mathrm{i}}$ is the same as in the most extensively studied regime, the ICRF. While the properties of the fast wave itself are qualitatively similar in the ICRF and the LHRF, important differences between wave damping in these two regimes arise from the interaction between the fast wave and the other cold plasma mode in these frequency ranges, which is known as the slow wave, the electron plasma wave, or the lower hybrid wave. In the ICRF, the slow wave can propagate only at very low densities $\omega<\omega_{\mathrm{pi}}$, so that interaction of the two waves is possible only very near the boundary of the plasma. The only role of the slow wave in ICRF fast wave experiments is thus as a possible parasitic loss process near the launching structure. ${ }^{5}$ By contrast, in the LHRF, slow and fast waves at the same frequency and at the same wavelength along the toroidal field can both propagate throughout most of the plasma volume. Any mechanism that would permit coupling between the two modes, which are actually completely distinct only in an unbounded uniform quiescent plasma, would be expected to have a strong effect on the absorption of wave power on electrons, because the electron Landau damping of the slow mode is much stronger than that of the fast wave at the same $n_{\|} \equiv k_{\|} c / \omega$. Hence, appreciable unlike-mode coupling would be expected to result in otherwise anomalously strong electron damping of the launched fast wave.

The electron damping of the fast wave has been calculated by several authors $\mathrm{s}^{2,6-8}$ in various limits. For a Maxwellian electron distribution, the most general result ${ }^{7}$ for the spatial damping rate due to electron damping may be written s $^{8}$

$$
2 \operatorname{Im}\left(k_{\perp}\right)=\operatorname{Re}\left(k_{\perp}\right) \frac{\sqrt{\pi}}{2} \beta_{\mathrm{e}} \xi e^{-\xi^{2}}\left[1+\frac{1}{\alpha^{2}}\right],
$$

where

$$
\begin{aligned}
\alpha & =\left(\frac{T_{\mathrm{e}}}{m_{\mathrm{i}} c^{2}}\right)\left(\frac{\omega^{2}-\Omega_{\mathrm{i}}^{2}}{\omega_{\mathrm{pi}}^{2}}\right)\left(S-n_{\|}^{2}\right)\left|K_{z z}\right|, \\
\xi & =\frac{\omega}{k_{11}} \sqrt{\frac{m_{\mathrm{e}}}{2 T_{\mathrm{e}}}} ; \quad \beta_{\mathrm{e}}=\frac{8 \pi n_{\mathrm{e}} T_{\mathrm{e}}}{B_{0}^{2}} ; \quad S=1-\frac{\omega_{\mathrm{pi}}^{2}}{\omega^{2}-\Omega_{\mathrm{i}}^{2}}+\frac{\omega_{\mathrm{pe}}^{2}}{\Omega_{\mathrm{e}}^{2}-\omega^{2}}, \\
K_{\mathrm{z} z} & =1+\frac{1}{k_{\| 1}^{2} \lambda_{\mathrm{D}^{2}}}[1+\xi Z(\xi)] .
\end{aligned}
$$

If $n_{\|}^{2} \ll|S| \simeq \omega_{\mathrm{pi}}^{2} /\left(\omega^{2}-\Omega_{\mathrm{i}}^{2}\right)$, and taking the cold plasma limit of $\left|K_{z z}\right|, \alpha$ reduces to

*Perhaps the first proposal ${ }^{4}$ for FWCD in a tokamak reactor was in this low frequency regime. 


$$
\alpha \simeq \frac{T_{\mathrm{e}}}{m_{\mathrm{e}} c^{2}}\left(\frac{\omega_{\mathrm{pi}}^{2}}{\omega^{2}}\right),
$$

so that at low frequencies, high density, and/or high electron temperature, $1 / \alpha^{2}$ is negligible compared to unity, and the damping length reduces to the well known formula

$$
2 \operatorname{Im}\left(k_{\perp}\right) \simeq \operatorname{Re}\left(k_{\perp}\right) \frac{\sqrt{\pi}}{2} \beta_{\mathrm{e}} \xi e^{-\xi^{2}}
$$

where the dispersion for the fast wave is very nearly $\operatorname{Re}\left(k_{\perp}\right) \simeq \omega / v_{\mathrm{A}}$. The overall toroidal magnetic field dependence of the damping is quite strong: $\operatorname{Im}\left(k_{\perp}\right) \propto B_{0}^{-3}$. The damping has a broad maximum around parallel phase velocities of $\xi=1 / \sqrt{2}$, or at a parallel index of refraction of

$$
n_{\|} \simeq \frac{23}{\sqrt{T_{\mathrm{e}}[\mathrm{keV}]}}
$$

At values of $n_{\|}$which can be launched easily with ordinary antenna structures with a directive spectrum, Eq. (3) shows that the electron temperature required for strong single pass electron absorption is much higher than has been achieved in tokamaks to date. The long damping length predicted by Eq. (1) at fairly low $n_{\|}$is the reason that the fast wave is well suited to driving current near the magnetic axis in a large tokamak reactor, while difficult to test in present day experimental devices. (Of course, the reverse applies to LHCD.) For the fast wave, the electron damping is generally too weak to produce a quasilinear flattening of the electron distribution with any reasonable fast wave power density, so that one cannot simultaneously access the efficient current drive regime of high phase velocities and maintain high single pass absorption. Therefore, the strategy employed in FWCD experiments generally has been to operate at the highest $n_{\|}$which can be launched with a given antenna structure and simultaneously to maximize the electron temperature and hence the single pass absorption with other forms of electron heating. Nonetheless, in every experiment performed to date, the single pass electron damping has been quite weak, so that multiple pass absorption is expected. Consequently, the relationship between the launched and absorbed spectrum is not as straightforward as in heating and current drive schemes with strong first pass absorption.

In the ICRF, ion cyclotron harmonic damping tends to dominate direct electron damping, so that various methods have been used to minimize this competing damping mechanism. At high aspect ratio, it may be possible to operate without any cyclotron harmonic layers in the plasma, or at least near the magnetic axis. For proof-of-principle experiments, operation at moderate cyclotron harmonics, $\omega=(3$ to 5$) \Omega_{\mathrm{i}}$ is adequate to avoid strong ion damping, as long as no significant source of energetic ions (NBI) exists in the plasma. Whether such a scenario is applicable in a reactor is unclear, due to harmonic damping on alphas. A practical difficulty in this frequency range stems from the high $n_{\|}$necessary for significant electron absorption: the antenna loading is relatively weak at high $n_{\|}$, due to the large evanescent region at the edge of the discharge for high $n_{\|}$. The low antenna loading can pose significant technical difficulties in launching high power levels.

By going towards higher frequencies, that is, towards the LHRF, some of these problems are alleviated. The $1 / \alpha^{2}$ term in Eq. (1) dominates, so that the fast wave damping at the same $n_{\|}$can be considerably stronger than in the low frequency limit [Eq. (2)]. Alternatively, one can maintain the same single pass absorption while 
improving the current drive efficiency by raising the frequency and operating at lower values of $n_{\|}$. By going to very high ion cyclotron harmonics, ion damping becomes negligible. For small tokamaks, the size of fast wave launching structures becomes relatively manageable at these frequencies. Finally, rf sources in this frequency range were already installed at several tokamak facilities by the early 1980s. For these reasons, the first tokarnak experiments on FWCD were performed in the LHRF.

\section{FWCD EXPERIMENTS IN THE LHRF}

By the mid 1980s, slow wave current drive in the LHRF was well established experimentally in tokamaks. ${ }^{9,10}$ The LHCD density limit scaling with frequency was well characterized experimentally ${ }^{9,11,12}$ though not completely understood theoretically. ${ }^{13,14}$ It was recognized that while both linear and nonlinear ion interaction can be avoided by operating with $\omega / \omega_{\mathrm{LH}} \gtrsim 2$, the relatively high $n_{\|}$necessary for accessibility to the center of a dense plasma would inevitably lead to strong Landau damping in the outer part of a large, hot $\left(T_{\mathbf{e}}(0) \gtrsim 15 \mathrm{keV}\right)$ reactor plasma. For slow and fast wave propagation in a slab geometry at the same frequency, the accessibility limit (due to mode conversion between the two modes with concomitant reflection) is identical for the two modes. However, the fast wave is unaffected by the lower hybrid resonance, so that fast waves can be used at lower frequencies $\left(\omega / \omega_{L H} \lesssim 1\right)$ for which the accessibility limit can be pushed down nearly to $n_{\|}=1$. In the tokamaks of the mid 1980s, the fast wave damping was very weak even in the LHRF, particularly at the low values of $n_{\|}$ that were practical to launch and at which the current drive efficiency was expected to be observably large. Therefore, though the driven current per dissipated wave power is independent of the launched wave mode, the observable efficiency (driven current per input wave power) was expected to be much lower for the fast wave than for the slow wave at the same frequency and $n_{\|}$. Thus, the signature of fast wiave current drive in the LHRF should be a weak direct electron interaction which should decrease only with power per particle as the density is raised through $\omega / \omega_{\mathrm{LH}}=2$ and then $\omega / \omega_{\mathrm{LH}}=1$.

In this context, the results from the experiments on FWCD in the LHRF were rather surprising. The first result reported ${ }^{15}$ was from JIPP T-IIU, using an $800 \mathrm{MHz}$ rf source. A four-element dipole antenna array with a double-layer Faraday shield was used to launch up to about $100 \mathrm{~kW}$ of $\mathrm{rf}$ power with a wide $n_{\|}$spectrum centered around $n_{\|} \sim 2$ for $90^{\circ}$ phasing. At the low density of $\bar{n} \simeq 3 \times 10^{12} \mathrm{~cm}^{-3}$, the current drive efficiency was observed to be identical to that observed with slow wave launch at the same frequency on JIPP T-IIU. However, the same density limit of about $\bar{n} \simeq 8 \times 10^{12} \mathrm{~cm}^{-3}$ was observed for both slow and fast wave current drive. Furthermore, the phenomenology around the density limit - the observation of fast ion tails correlated the presence of parametric decay into ion-cyclotron quasimodes, where the cyclotron frequency of the decay waves corresponds to the field at the outboard midplane of the discharge - were similar for the two launched modes. Interestingly, the maximum electron interaction occurred at antenna phasing of $0^{\circ}$, and the decrease in electron interaction as the phase angle was increased was independent of the relative sign of the phase velocity and the direction of the plasma current. The JIPP T-IIU experimentalists speculated that the parametric decay resulted from mode conversion from the fast to the slow wave, and that the density limit therefore was due to channeling of wave energy into the ion tail via the parametric decay of the slow waves. They did not suggest, however, the possibility that the efficient current drive at lower densities was also due to damping of mode-converted slow waves. This possibility was mentioned in another paper ${ }^{16}$ from JIPP T-IIU, which will be described later in this review. 
Two different fast wave launching structures were compared in a set of experiments ${ }^{17,18}$ on the Princeton Large Torus (PLT). These experiments were also performed at $800 \mathrm{MHz}$. In the first set of experiments, an array of six small loop antennas, ${ }^{19}$ with no Faraday shield, was used to launch up to $150 \mathrm{~kW}$ for up to $0.3 \mathrm{sec}$. A second set of experiments used a $4 \times 3$ (toroida' $\times$ poloidal) array of dielectric-loaded waveguides to launch up to $340 \mathrm{~kW}$ of power. The coupling properties of the latter launching structure were studied in some detail theoretically ${ }^{20,21}$ and experimentally. ${ }^{21}$ The conclusion of the coupling study was that under the conditions used in the current drive experiments, only a small fraction (less than $5 \%$ for $180^{\circ}$ phasing) of the launched power coupled directly to the slow wave, with the remainder being launched in the desired fast wave polarization. Yet for both the dielectric-loaded array coupler and the array of loop antennas the current drive efficiency was similar to the $800 \mathrm{MHz}$ slow wave couplers already studied on PLT, ${ }^{22}$ and the density limit for fast electron interaction was independent of the launched mode (Fig. 1). A detailed comparison of the $800 \mathrm{MHz}$ slow and fast wave experiments concentrated on the phenomena associated with the density limit; no significant difference between the two launches was found.

Another detailed comparison of slow and fast wave launch at the same frequency on the same device was performed on the JFT-2M tokamak. ${ }^{23}$ In this experiment, fast waves launched with a phased pair of loop antennas were compared with slow waves launched from a pair of waveguides, both at $750 \mathrm{MHz}, P_{\mathrm{r}:}=100 \mathrm{~kW}$. While the density limit for observable current drive was again identical for the two launches, the details of the phenomena just below the density limit were slightly different. Parametric decay activity was weaker for the fast wave launch in this density regime, and the maximum density for which a fast electron tail was observed was somewhat higher. It was concluded that at low density slow waves were responsible for the observed current drive, and that the small effect of FWCD was manifest only in the neighborhood of the density limit.

An explanation of these results was proposed by the present author, ${ }^{18}$ based on ideas mentioned by Ando et al. (Ref. 16) and Andrews. ${ }^{24,25}$ The fact that the fast wave electron damping is so much weaker than that of the slow wave at the same $n_{\|}$ implies that any power which is undergoing mode conversion back and forth between the two modes is much more likely to be absorbed as a slow wave than as a fast wave. ${ }^{16}$ Furthermore, a simple statistical argument ${ }^{18}$ shows that even without damping, given a random mode coupling mechanism, wave power launched as a fast wave will end up in the slow wave polarization, while the reverse is not true. The net result of these two phenomena is that wave power launched in the LHRF in either of the two polarizations is likely to be absorbed as a slow wave, and hence be subject to the slow wave density limit. All that is necessary for this argument to apply is the existence of a random mode coupling mechanism.

Two such mechanisms important in different regimes were first discussed in detail by Bonoli and $\mathrm{Ott}^{26}$ in the context of slow wave launch. In a quiescent toroidal plasma with inverse aspect ratio $\gtrsim 0.2$, ray trajectories starting at $n_{\|}$just above the critical $n_{\|}$for accessibility in a slab geometry become ergodic. The resultant random shifting up and down of $n_{\|}$along the ray trajectory along with the accompanying mode conversion constitutes a random mode coupling mechanism to which the statistical argument mentioned above applies. The other mode coupling mechanism applies even in a slab geometry: in a turbulent plasma in which the density fluctuation spectrum 
has significant power in the range of $k_{\perp}$ equal to the difference in $k_{\perp}$ of the two wave modes near the accessibility limit, unlike mode scattering ${ }^{24}$ is possible, and as shown by Andrews, ${ }^{25}$ is likely to be significant in the edge region of tokamaks for wave launching in the LHRF.

At high central densities such that $\omega \ll \omega_{L H}(\rho=0)$, mode coupling is not possible near the center of the discharge, since the slow wave cannot propagate there. From this perspective, the essential difference between the FW experiments in the LHRF and those in the ICRF is the fraction of the plasma volume in which both modes can propagate. In the ICRF, this region of overlap can vanish for $n_{\|}$high enough to obtain significant electron damping, because the FW cutoff density can be higher than the lower hybrid resonance density. Thus, at low frequency, wave power can propagate in the core of the discharge only as either a fast wave or an ion Bernstein wave. Hence, FWCD at low frequency will not be subject to a density limit related to wave accessibility.

For this reason, along with the fact that the high values of $n_{\|}$necessary for reasonable single pass electron damping can be launched with large antenraa structures more conveniently at low frequencies, FWCD experiments in the LHRF have been largely abandoned in favor of the ICRF in recent years. Work with fast wave launch in the LHRF has been carried on the Versator II tokamak at MIT, where FW launch with a slotted waveguide coupler at $2.45 \mathrm{GHz}$ has been studied, ${ }^{27}$ and a detailed experimental study of parametric decay activity in the LHRF in which $800 \mathrm{MHz}$ slow wave, $2.45 \mathrm{GHz}$ slow wave, and $800 \mathrm{MHz}$ fast wave launch (the latter using a four element array of dielectric loaded waveguides) have been compared on the same machine. ${ }^{28}$ From the point of view discussed above, one would expect for the fast wave launch, the mode converted power in the edge region would tend to raise the energy density in that region, and hence lower the threshold power for the parametric decay instability. In the experiment, parametric decay and associated edge electron heating with the remarkably low power threshold of about $P_{\mathrm{rf}}=50 \mathrm{~W}$ are reported.

\section{EXPERIMENTS ON DIRECT ELECTRON DAMPING OF THE ICRF FW}

The first set of experiments on FW direct electron interaction in the ICRF were intended to establish a connection between the damping theory discussed in the introduction and experimental results, as a first step in the development of FWCD in this frequency regime. To make the electron damping significant, the highest $n_{\|}$that can be launched must be used. Therefore the antenna phasing used for the multiple loop arrays in these experirnents is generally $180^{\circ}$, which coincidentally is also the most favorable antenna phasing for minimizing impurities. ${ }^{1}$ While operating at low frequencies raises $n_{\|}$for a given antenna geometry, Eq. (1) shows that the damping increases with frequency at a fixed $n_{\|}$, through $\operatorname{Re}\left(k_{\perp}\right) \propto f$ and $1 / \alpha^{2} \propto f^{4}$ in the cold plasma limit. Furthermore, operating at higher frequency helps to minimize ion cyclotron damping, which otherwise dominates. Hence these experiments tend to be done at the upper end of the available frequency range at each tokamak facility, and at low toroidal field [Eq. (1) shows the damping increases with decreasing toroidal field as $B_{0}^{-3}$.

The first detailed experimental study of FW direct electron absorption in this regime was carried out on the JFT-2M tokamak at $200 \mathrm{MHz}$. After a preliminary experiment ${ }^{23}$ using the same two-loop antenna as had been used at $750 \mathrm{MHz}$, a new four-strap antenna and $4 \times 200 \mathrm{~kW} 200 \mathrm{MHz}$ rf system were constructed. In the first set of results reported from this system, ${ }^{29}$ the lower hybrid resonance layer for $200 \mathrm{MHz}$ was located at $r / a=0.93$; clearly, slow waves can have nothing to do with 
the central electron heating reported there, though parametric decay of mode converted slow waves could suill be an important energy loss mechanism. Central electron heating was observed only for $\xi \leqslant 4$. A more detailed study of electron absorption ${ }^{30}$ performed by the same group employed a wider range of discharge conditions and used a FW amplitude modulation technique to deduce the radial deposition profile. The experimentally determined deposition profile was found to be strongly centrally peaked, in accordance with theoretical expectations (Fig. 2). Both the modulation technique and analysis of the rate of change of the plasma stored energy at FW turn-on and turn-off yielded an overall absorption efficiency of about 0.3 to 0.4 in the best case. It is claimed that this absorption coefficient is consistent with ray-tracing calculations of single pass absorption. These authors speculated that the power not absorbed on the first pass is collisionally dissipated in the edge plasma and/or the vacuum vessel walls; this view is supported by the observation of an anti-correlation between absorption efficiency and impurity influx.

A series of discharges in the JET tokamak with ICRF heating in a number of heating regimes were analyzed by Eriksson and Hellsten to determine the relative contributions of direct electron absorption and slowing down of an ion tail produced by ICRH to the observed electron heating. ${ }^{31}$ In these cases, a further complication is that direct electron heating can result from either damping of the fast wave or of ion Bernstein waves (IBWs) produced by mode conversion. A painstaking analysis of the time dependence of the electron temperature enabled these authors to distinguish between the different components of electron heating. In cases with high minority concentrations, discharges where the electron heating is primarily due to direct electron absorption were identified. With $180^{\circ}$ phasing of the two-element antenna arrays, the high $n_{\|}$tends to minimize the contribution of mode converted IBWs and maximize direct electron absorption of the fast wave itself. With $0^{\circ}$ antenna phasing, mode conversion becomes important, and the measured electron deposition profile becomes strongly peaked around the mode conversion layer.

A more recent set of experiments on JET intended to maximize direct electron absorption of the fast wave were reported by Start et $a .^{32}$ In contrast to the earlier JET experiment, here the lowest order ion cyclotron resonance layer was displaced inboard of the magnetic axis to create a substantial central volume of the plasma free of any cyclotron resonances. A square-wave modulation of the rf power was employed to measure the direct electron absorption in this central region from time dependent analysis of $T_{e}(t)$. In this situation, most of the power not absorbed on the first pass through the center is absorbed at the $\omega=2 \Omega_{\mathrm{H}}$ layer on the high field side of the magnetic axis. Measurements showed approximately $22 \%$ of the input if power was directly absorbed by electrons in the central region, in good agreement with full wave and ray tracing codes.

Some recent experiments in TFTR ${ }^{33}$ at $47 \mathrm{MHz}$ have explored similar regimes of FW direct damping as the JET work. Similar amplitude modulation techniques were used to study direct electron absorption in two regimes. In one regime $\left(B_{\mathrm{T}}=2.3 \mathrm{~T}\right)$, the second harmonic of the majority ${ }^{3} \mathrm{He}$ passed through the center of the discharge; approximately $30 \%$ to $50 \%$ of the power appeared to be absorbed on electrons near the center of the discharge. In the other regime studied in detail, the fundamental resonance of ${ }^{3} \mathrm{He}$ passed through the center $\left(B_{\mathrm{T}}=4.8 \mathrm{~T}\right)$, but no ${ }^{3} \mathrm{He}$ was introduced into the deuterium plasma. In this case, about $60 \%$ of the rf power was absorbed by electrons near the center of the of the plasma. This discharge was in the 'supershot' 
regime, and reached a central electron temperature of about $9 \mathrm{keV}$ with strong NB heating $\left(P_{\mathrm{NB}}=24 \mathrm{MW}\right)$.

Two sets of experiments on the JIPP T-IIU tokamak on direct electron absorption of the FW in the ICRF have been reported. The first experiment ${ }^{16}$ was performed at $40 \mathrm{MHz}$ and at very low toroidal field so that $\omega \sim 13 \Omega_{\mathrm{H}}$. In this case, the plasma was produced by the rf power alone. A phased array of five loop antennas was used to launch fast waves with $n_{\|} \approx 7$ for $\Delta \phi=90^{\circ}$, at up to $400 \mathrm{~kW}$. Though the direction of the current was determined by the polarity of the vertical field, as is often the case in startup experiments, the efficiency of the current drive did respond to the antenna phase angle roughly as expected. The electron temperature in this experiment did not exceed about $10 \mathrm{eV}$, so that the remarkable feature of this experiment was that any electron interaction was observed at all; under these conditions, $\xi \sim 23$, so direct electron absorption of the nominal launched $n_{\|}$would be expected to be utterly negligible.

In the more recent experiments ${ }^{34}$ on JIPP T-IIU, a $130 \mathrm{MHz}$ if system was used to power a four-element array of loop antennas mounted on the high field side of the torus. In this case, $\Delta \phi=180^{\circ}, n_{\|}= \pm 5$. The single pass absorption was predicted to be less than $0.1 \%$ for the experimental conditions. Conventional theories indicate that mode conversion to the IBW should be negligible. Nonetheless, significant electron heating was observed along with a tail in the deuterium neutral particle charge exchange spectrum. It was speculated that these results might be related to anomalously strong mode conversion near $\omega=6 \Omega_{\mathrm{D}}$ and subsequent absorption of the IBW near the $\omega=5 \Omega_{\mathrm{D}}$ resonance. The net absorption efficiency was estimated to be $\gtrsim 50 \%$ despite the very low single pass absorption.

Another tokamak experiment in which unexpectedly strong absorption of the FW was observed has been carried out on the Phaedrus-T device. ${ }^{35}$ Here a two-strap antenna was operated at $19 \mathrm{MHz}$, so that with $\Delta \phi=90^{\circ}$, the peak of the vacuum spectrum was $n_{\|}=26$, and $\xi<1$ for the peak of the vacuum spectrum. The antenna loading to these very short wavelengths however is very weak, so that the value of $\xi$ characterizing the power that actually propagates in the plasma would be expected to be significantly larger. The expected weak damping was evident from the eigenmodes observed on both the antenna loading and on magnetic probes in the edge plasma as the density rose during high power $\mathrm{rf}$ injection. Nonetheless, the damping length measured in the edge plasma with magnetic probes appeared to be several times shorter than expected. Weak electron heating near the center of the discharge was observed for $90^{\circ}$ antenna phasing, while no heating was found with $180^{\circ}$ phasing, probably as a result of the extremely weak coupling to the high $n_{\|}$excited by the latter phasing.

A recent experimental study ${ }^{36,37}$ of direct electron damping on the DIII-D tokamak used a four-element phased antenna array and a $2 \mathrm{MW} 60 \mathrm{MHz}$ if source. For the damping experiments $180^{\circ}$ phasing was used to excite $n_{\|}$in the neighborhood of 10 . The upshift of $n_{\|}$due to finite aspect ratio ( $R n_{\|}=$constant) is important in the relatively low aspect ratic DIII-D device: $n_{\|}(\rho=0)=n_{\|} \operatorname{lant}_{1}\left(R_{0}+a\right) / R_{0}$

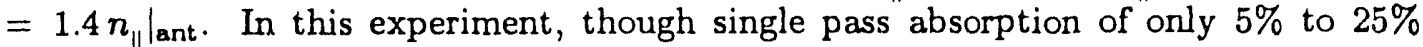
was calculated using measured profiles, about $80 \%$ to $100 \%$ of the coupled rf power was absorbed (Fig. 3). Evidently efficient multiple pass absorption must occur. The central electron heating effectiveness was found to depend strongly on $\xi$ evaluated at the center of the discharge: large increases in $T_{e}(0)$ were observed only if $\xi \lesssim 1.5$. The deposition profile was studied by modulation techniques similar to those employed in the previous experiments. A highly peaked deposition profile was found, in agreement 
with the predictions of a full-wave code. A steady-state ELMing $\mathrm{H}$-mode was produced with FW direct electron heating as the sole auxiliary heating source. The fact that the power threshold for the $\mathrm{H}$-mode transition was approximately the same as for $\mathrm{H}$-modes produced by ECH or NBI implies that nearly $100 \%$ of the fast wave power must be absorbed in the discharge.

Assuming that the fundamental theory is correct, the good absorption generally observed in the experiments even when the predicted single pass absorption is very poor implies that multiple pass absorption must occur. Though for heating purposes, multiple pass absorption should not present a problem as long as $\mathrm{rf} /$ wall interactions are minimized, such a large number of passes may imply loss of control over the absorbed spectrum. For efficient current drive, then, good single pass absorption might still be necessary. This fact, along with the formidable technical problems associated with launch of a high $n_{\|}$spectrum with good directivity, makes FWCD experiments in the ICRF the most challenging of the experiments discussed in this review.

\section{FWCD EXPERIMENTS IN THE ICRF}

Before successful experiments on FWCD in the ICRF could be performed, solutions had to be found for the aforementioned technical challenges. A complete discussion of the work that has been carried out towards the solution of these problems is beyond the scope of this review; this effort has been described in some of the references. ${ }^{38-40}$ Continuing work in this area is discussed in several papers in these proceedings.

A consequence of these technical problems has been that until recently, launching of significant ICRF power with a directive spectrum peaked at a value of $\left|n_{\|}\right|$high enough to yield single pass damping in even the $5 \%$ to $10 \%$ range has been extremely difficult. Therefore, no conclusive evidence of substantial current driven by direct electron absorption of ICRF fast waves was obtained prior to the DIII-D results described in Ref. 41. The same four-element antenna array that had been used for the DIII-D FW direct electron heating studies was used for the FWCD experiments; in the latter case, antenna phasing of $(0, \pi / 2, \pi, 3 \pi / 2)$ was used to launch a directive spectrum peaked between $n_{\|}=5$ to 7 at $60 \mathrm{MHz}$. Up to $1.1 \mathrm{MW}$ was coupled with this phasing into relatively low density discharges $\left(\bar{n}_{\mathrm{e}} \sim 1 \times 10^{13} \mathrm{~cm}^{-3}\right)$. Preheating with $60 \mathrm{GHz} \mathrm{ECH}$ ( $2 \Omega_{\mathrm{e}}, \mathrm{X}$-mode) was used to raise the single pass absorption of the FW to about $10 \%$. Studies of the global confinement properties of the discharges in which ECH and FW with directive antenna phasing were compared again indicated that nearly $100 \%$ of the FW power was absorbed, evidently by multiple passes of the FW through the hot core of the plasma.

In the best case with EC preheating, up to $160 \mathrm{kA}$ of the $400 \mathrm{kA}$ total plasma current were sustained noninductively, while without preheating, $135 \mathrm{kA}$ of noninductive current were observed. In both cases, $40 \mathrm{kA}$ of the noninductive current could be attributed to the neoclassical bootstrap current. The current drive efficiency was found to increase roughly linearly with the central electron temperature (Fig. 4). The observed current drive efficiency in the customary units could be expressed as $\gamma \equiv \bar{n}_{\mathrm{e}} I_{\mathrm{rf}} R_{0} / P_{\mathrm{FW}} \simeq 5.5 \times 10^{17} T_{\mathrm{e}}(0)[\mathrm{keV}] \mathrm{A} / \mathrm{W} / \mathrm{m}^{2}$, with the best result obtained to date being $\gamma \simeq 0.020 \times 10^{20} \mathrm{~A} / \mathrm{W} / \mathrm{m}^{2}$, obtained at a central $T_{\mathrm{e}}(0) \simeq 3 \mathrm{keV}$. The increase in the neoclassical bootstrap current caused by the rf has been subtracted from the noninductive current to obtain this number. Higher of power levels are expected to produce higher electron temperatures, and hence higher current drive efficiencies 
in the near future. FWCD modified the magnetic structure (sawtooth behavior) near the magnetic axis, with quite different effects observed for counter-FWCD than for co-FWCD. A strong effect of this kind is expected as a result of the highly centrallypeaked FW deposition profile. Generally, the DIII-D FWCD results are consistent with multiple pass ray tracing calculations, as well as with full-wave code calculations. ${ }^{42}$

\section{CONCLUSIONS AND OUTLOOK}

Tokamak experiments on electron heating and current drive by direct electron absorption of fast waves have made significant advances in the last eight years. The results of early experiments with fast waves in the lower hybrid range of frequencies can be understood in terms of mode conversion of the launched fast waves to lower hybrid slow waves and subsequent efficient absorption of the slow waves. Direct electron absorption experiments in the ICRF have demonstrated that this technique of heating electrons is a useful addition to the already well established ICRF plasma heating methods of minority and second harmonic heating. Noninductive currents in excess of $0.1 \mathrm{MA}$ have been driven by the absorption of fast waves with a directive spectrum and the predicted favorable temperature scaling of the FWCD efficiency has been observed.

In the next few years, this favorable temperature scaling alorig with improved solutions to the technical problems of directive FW launch should permit extension of the FWCD regime to the $\sim 1 \mathrm{MA}$ level, with complete sustainment of the plasma current by fast wave power alone. An upgrade of the DIII-D FWCD system to $6 \mathrm{MW}$ in the 30 to $120 \mathrm{MHz}$ range is in progress; with additional electron heating, driven currents in the 0.5 to $1.0 \mathrm{MA}$ range are expected. On JFT-2M, increased ECH power and improved $200 \mathrm{MHz}$ FW antennas should permit unambiguous observation of FWCD in the next two years. New four-element antenna arrays are being installed in JET at this writing; these antennas along with an improved phase control system will enable FWCD experiments at truly reactor relevant power levels (up to $32 \mathrm{MW}$ of generated power) and central electron temperatures. Other tokamaks for which FWCD experiments have been discussed include Tore Supra, JT-60U, TFTR, and Alcator C-Mod. In the next few years, FWCD should be further validated by these near-term experiments and will therefore become the central current drive method of choice for future devices as tokamak plasmas become more reactor-like. Indeed, this is the environment in which fast wave current drive has always been expected to operate most effectively.

\section{ACKNOWLEDGMENT}

This is a report of work sponsored by the U.S. Department of Energy under Contract No. DE-AC03-89ER51114.

\section{REFERENCES}

1. J.-M. Noterdaeme, Radio-Frequency Power in Plasmas: Ninth Topical Conference, Charleston, SC 1991 (American Institute of Physics, New York, 1992), p. 71.

2. T.H. Stix, Nucl. Fusion 15, 737 (1975).

3. J.G. Cordey et al., in Proceedings of the 18th European Conference on Controlled Fusion and Plasma Physics, (Berlin, 1991) 15C, Part III, (EPS, 1991), p. 385.

4. D.J.H. Wort, Plasma Phys. 13, 258 (1971).

5. E.A. Berro and G.J. Morales, IEEE Trans. Plas. Sci. 18, 142 (1990). 
6. D. Moreau, M.R. O'Brien, M. Cox, and D.F.H. Start, in Proceedings of the 14th European Conference on Controlled Fusion and Plasma Physics, (Madrid, 1987) 11D, Part III, (EPS, 1987) p. 1007.

7. S.C. Chiu, V.S. Chan, R.W. Harvey, and M. Porkolab, Nucl. Fusion 29, 2175 (1989).

8. M. Porkolab, Radio-Frequency Power in Plasmas: Ninth Topical Conference, Charleston, SC 1991 (American Institute of Physics, New York, 1992), p. 197.

9. W. Hooke, Plasma Phys. and Contr. Fusion 26, Vol. 1A, 133 (1984).

10. N.J. Fisch, Rev. Mod. Phys. 59, 175 (1987).

11. F. Alladio, E. Barbato, G. Bardotti, et al., Nucl. Fusion 24, 725 (1984).

12. M.J. Mayberry, M. Porkolab, K.-I. Chen, et al., Phys. Rev. Lett. 55, 829 (1985).

13. J.-G. Wegrowe and F. Engelmann, Comments Plasma Phys. and Contr. Fusion 8, 211 (1984).

14. V.S. Chan and C.S. Liu, Fusion Technol. 7, 288 (1985).

15. K. Ohkubo, Y. Hamada, Y. Ogawa, et al., Phys. Rev. Lett. 56, 2040 (1986).

16. R. Ando, E. Kako, Y. Ogawa and T. Watari, Nucl. Fusion 26, 1619 (1986).

17. R.I. Pinsker, P.L. Colestock, S. Bernabei, et al., Applications of Radio-Frequency Power to Plasmas: Seventh Topical Conference, Kissimmee, FL 1987 (American Institute of Physics, New York, 1987), p. 175.

18. R.I. Pinsker, Fast Wave Current Drive Experiments on the Princeton Large Torus, Ph.D. dissertation, Princeton University (1988).

19. P.L. Colestock, J.E. Stevens, J.C. Hosea, et al., RF Plasma Heating: Sixth Topical Conference, Callaway Gardens, GA 1985 (American Institute of Physics, New York, 1985), p. 48.

20. R.I. Pinsker, R.E. Duvall, C.M. Fortgang and P.L. Colestock, Nucl. Fusion 26, 941 (1986).

21. R.I. Pinsker and P.L. Colestock, Nucl. Fusion 32, 1789 (1992).

22. J.E. Stevens, R.E. Bell, S. Bernabei, et al., Nucl. Fusion 28, 217 (1988).

23. Y. Uesugi, T. Yamamoto, K. Hoshino, et al., in Proceedings of the 14th European Conference on Controlled Fusion and Plasma Physics (Madrid, 1987), 11D, Part III (EPS, 1987) p. 942.

24. P.L. Andrews, Phys. Rev. Lett. 54, 2022 (1985).

25. P.L. Andrews, Bull. Am. Phys. Soc. 32, 1793 (1987).

26. P.T. Bonoli and E. Ott, Phys. Fluids 25, 359 (1982).

27. J.A. Colborn, R.R. Parker, S.C. Luckhardt, et al., Nucl. Fusion 31, 960 (1991).

28. J. Villaseñor, M. Porkolab, et al., these proceedings.

29. T. Yamamoto, Y. Uesugi, H. Kawashima, et al., Phys. Rev. Lett. 63, 1148 (1989).

30. Y. Uesugi, T. Yamamoto, H. Kawashima, et al., Nucl. Fusion 30, 831 (1990).

31. L.-G. Eriksson and T. Hellsten, Nucl. Fusion 29, 875 (1989).

32. D.F.H. Start, D.V. Bartlett, V.P. Bhatnagar, et al., Nucl. Fusion 30, 2170 (1990).

33. J.R. Wilson, M.G. Bell, H. Biglari, et al., "ICRF Heating on TFTR - Effect on Stability and Performance," presented at 14th Int. Conf. on Plasma Phys. and Controlled Fusion Research, Würzburg, Germany, 1992, paper IAEA-CN-56/E-2-2.

34. Y. Takase, T. Watari, R. Kumazawa, et al., Nucl. Fusion 30, 1585 (1990).

35. N. Hershkowitz, R. Majeski, P. Probert, et al., Radio-Frequency Power in Plasmas: Ninth Topical Conference, Charleston, SC 1991 (American Institute of Physics, New York, 1992), p. 267. 
36. C.C. Petty, R.I. Pinsker, M.J. Mayberry, et al., Radio-Frequency Power in Plasmas: Ninth Topical Conference, Charleston, SC 1991 (American Institute of Physics, New York, 1992), p. 96.

37. C.C. Petty, R.I. Pinsker, M.J. Mayberry, et al., Phys. Rev. Lett. 69, 289 (1992).

38. F.W. Baity et al., in Fusion Technology 1990 (Proc. 16th Symp., London, 1990) (North-Holland, Amsterdam, 1991), Vol. 2, p. 1035.

39. R.I. Pinsker et al., Proc. of 14th IEEE/NPSS Symp. on Fusion Engineering (San Diego, CA, 1991) (IEEE, Piscataway, NJ, 1992), Vol. 1, p. 115.

40. R.H. Goulding et al., Radio-Frequency Power in Plasmas: Ninth Topical Conference, Charleston, SC 1991 (American Institute of Physics, New York, 1992), p. 287.

41. R.I. Pinsker, C.C. Petty, M. Porkolab, F.W. Baity, et al., "Direct Electron Heating and Current Drive with Fast Waves in DIII-D," presented at 14th Int. Conf. on Plasma Physics and Controlled Fusion Research, Würzbu , Germany, 1992, paper IAEA-CN-56/E-2-4.

42. P.T. Bonoli, "Status and Comparison of Codes Used for Fast Wave Current Drive," these proceedings.

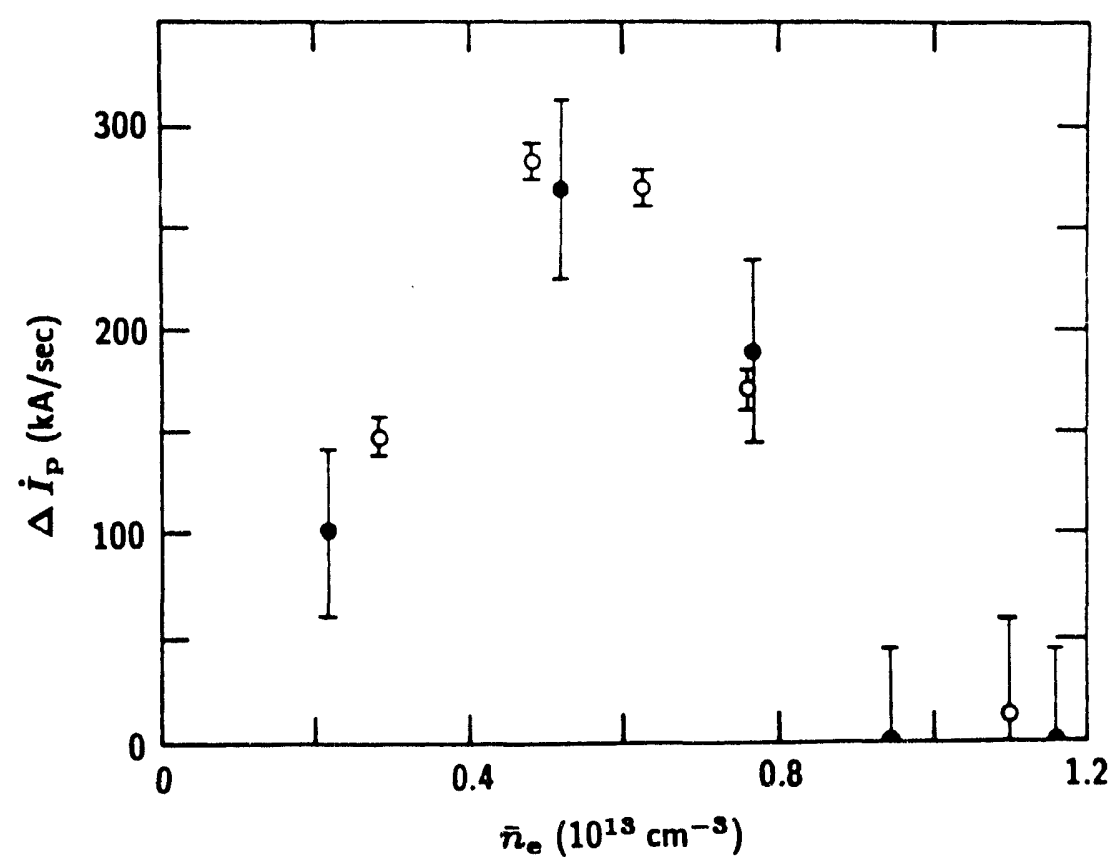

Fig. 1. Comparison of the change in the decay rate of the plasma current as a function of line-averaged density for two $800 \mathrm{MHz}$ couplers on PLT: $\circ=$ six-waveguide slow wave array, $\bullet=4 \times 3$ dielectricloaded waveguide fast wave launcher. This quantity is a rough measure of the noninductively driven current (Ref. 17). 

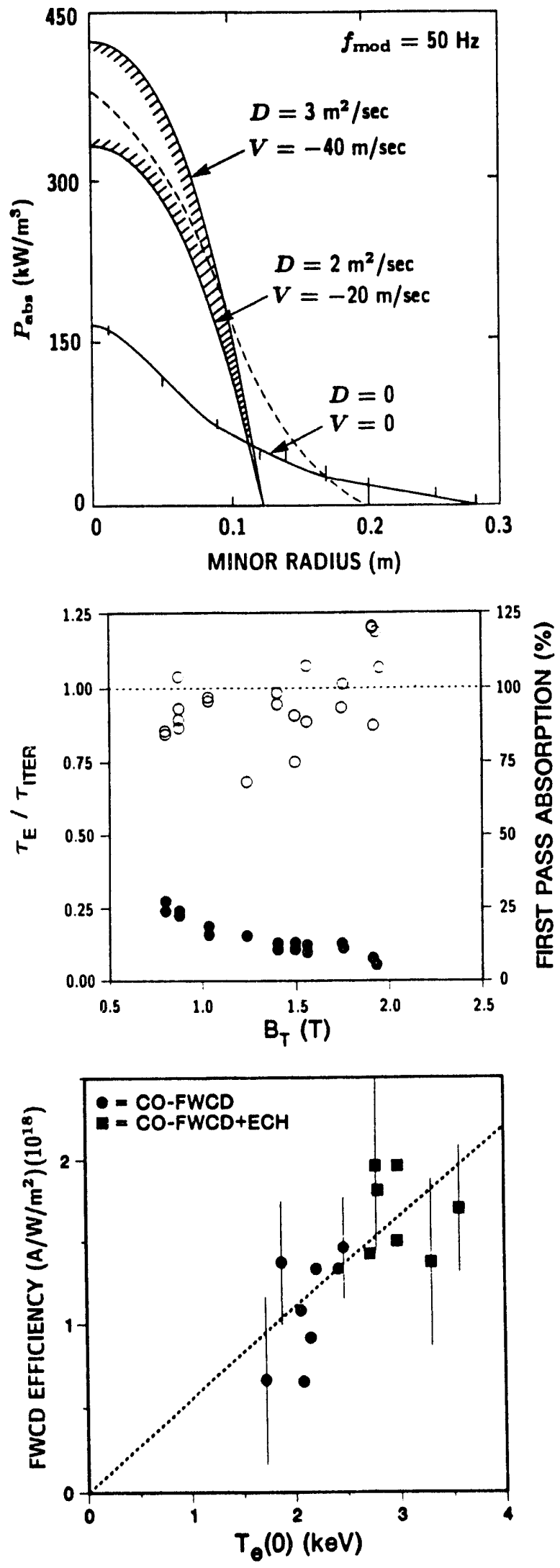

Fig. 2. $200 \mathrm{MHz}$ FW power deposition profile measured in JFT-2M with a modulation technique (shaded region) and calculated from single pass ray tracing (dashed curve) (from Ref. 30).

Fig. 3. The measured global absorption efficiency in DIII-D (o) with $60 \mathrm{MHz}$ FW direct electron heating as a function of toroidal field. Also shown is the calculated first-pass absorption (•) (Ref. 37).

Fig. 4. $60 \mathrm{MHz}$ FWCD efficiency measured in DIII-D with preheating (squares) and without (circles), as a function of the central electron temperature. Also indicated is the bestfitting line passing through the origin, with a slope of $5.5 \times 10^{17} \mathrm{~A} / \mathrm{W} / \mathrm{m}^{2} / \mathrm{keV}$ (Ref. 42). 

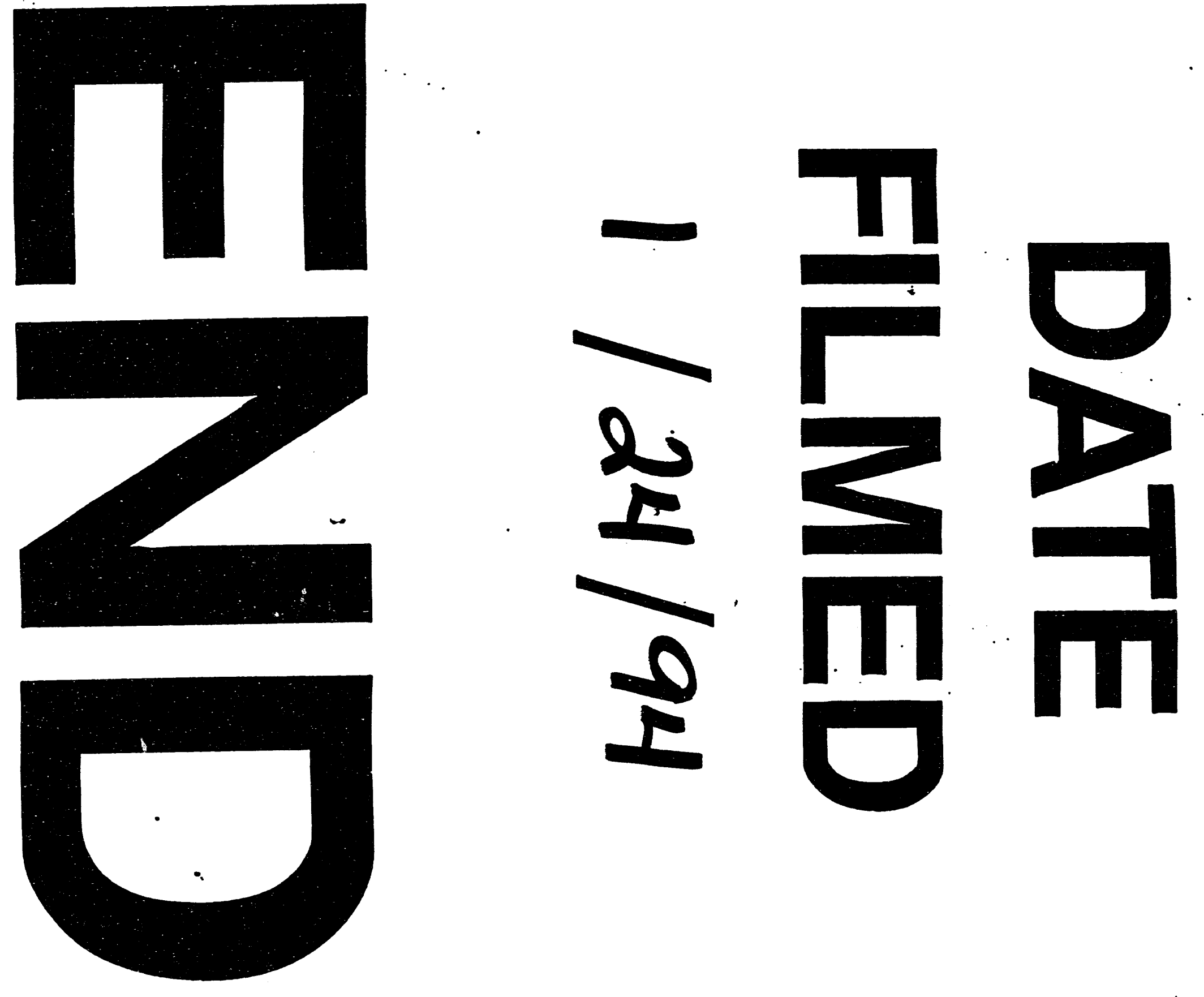
